\title{
Stellar Multiplicity Meets Stellar Evolution and Metallicity: The APOGEE View
}

\author{
Carles Badenes ${ }^{1,2}$ (10) Christine Mazzola ${ }^{1}$, Todd A. Thompson ${ }^{3}$, Kevin Covey ${ }^{4}$ (1), Peter E. Freeman ${ }^{5}$, Matthew G. Walker ${ }^{6}$ (1), \\ Maxwell Moe ${ }^{7,21}$, Nicholas Troup ${ }^{8,9}$ (1), David Nidever ${ }^{10}$ (D) , Carlos Allende Prieto ${ }^{11}$, Brett Andrews ${ }^{1}$ (D), Rodolfo H. Barbá ${ }^{12}$, \\ Timothy C. Beers ${ }^{13}\left(\mathbb{D}\right.$, Jo Bovy ${ }^{14,22}$ (), Joleen K. Carlberg ${ }^{15}$, Nathan De Lee ${ }^{16,17}$, Jennifer Johnson ${ }^{3}$, Hannah Lewis ${ }^{8}$, \\ Steven R. Majewski ${ }^{8}$ (10, Marc Pinsonneault ${ }^{3}$ (i), Jennifer Sobeck ${ }^{18}$, Keivan G. Stassun ${ }^{17}$ (1), \\ Guy S. Stringfellow ${ }^{19}$ (i), and Gail Zasowski ${ }^{20}$ \\ ${ }^{1}$ Department of Physics and Astronomy and Pittsburgh Particle Physics, Astrophysics and Cosmology Center (PITT PACC), University of Pittsburgh, 3941 O’Hara \\ Street, Pittsburgh, PA 15260, USA; badenes@ pitt.edu \\ ${ }^{2}$ Institut de Ciències del Cosmos (ICCUB), Universitat de Barcelona (IEEC-UB), Martí Franqués 1, E08028 Barcelona, Spain \\ ${ }^{3}$ Department of Astronomy and the Center for Cosmology and Astro-Particle Physics, The Ohio State University, Columbus, OH 43210, USA \\ ${ }_{5}^{4}$ Department of Physics and Astronomy, Western Washington University, Bellingham, WA 98225, USA \\ ${ }^{5}$ Department of Statistics, Carnegie Mellon University, 5000 Forbes Avenue, Pittsburgh, PA 15213, USA \\ ${ }^{6}$ McWilliams Center for Cosmology, Department of Physics, Carnegie Mellon University, 5000 Forbes Avenue, Pittsburgh, PA 15213, USA \\ ${ }^{7}$ Steward Observatory, University of Arizona, 933 North Cherry Avenue, Tucson, AZ 85721, USA \\ ${ }^{8}$ Department of Astronomy, University of Virginia, Charlottesville, VA 22904-4325, USA \\ ${ }^{9}$ Department of Physics, Salisbury University, Salisbury, MD 21801, USA \\ ${ }^{10}$ National Optical Astronomy Observatory, 950 North Cherry Avenue, Tucson, AZ 85719, USA \\ ${ }^{11}$ Instituto de Astrofísica de Canarias, Vía Láctea, E-38205 La Laguna, Tenerife, Spain \\ 12 Departamento de Física y Astronomía, Av. Juan Cisternas 1200 Norte, Universidad de La Serena, La Serena, Chile \\ ${ }^{13}$ Department of Physics and JINA Center for the Evolution of the Elements, University of Notre Dame, Notre Dame, IN 46556, USA \\ ${ }^{14}$ Department of Astronomy and Astrophysics and Dunlap Institute for Astronomy and Astrophysics, University of Toronto, \\ 50 St. George Street, Toronto, Ontario M5S 3H4, Canada \\ ${ }^{15}$ Space Telescope Science Institute, 3700 San Martin Drive, Baltimore, MD 21218, USA \\ ${ }^{16}$ Department of Physics, Geology, and Engineering Tech, Northern Kentucky University, Highland Heights, KY 41099, USA \\ ${ }^{17}$ Department of Physics and Astronomy, Vanderbilt University, Nashville, TN, USA \\ ${ }^{18}$ Department of Astronomy, Box 351580, University of Washington, Seattle, WA 98195, USA \\ ${ }^{19}$ Center for Astrophysics and Space Astronomy, Department of Astrophysical and Planetary Sciences, University of Colorado, Boulder, CO 80309, USA \\ ${ }^{20}$ Department of Physics and Astronomy, University of Utah, Salt Lake City, UT 84112, USA \\ Received 2017 October 31; revised 2018 January 10; accepted 2018 January 12; published 2018 February 21
}

\begin{abstract}
We use the multi-epoch radial velocities acquired by the Apache Point Observatory Galactic Evolution Experiment (APOGEE) survey to perform a large-scale statistical study of stellar multiplicity for field stars in the Milky Way, spanning the evolutionary phases between the main sequence (MS) and the red clump. We show that the distribution of maximum radial velocity shifts $\left(\Delta \mathrm{RV}_{\max }\right)$ for APOGEE targets is a strong function of $\log g$, with MS stars showing $\Delta \mathrm{RV}_{\max }$ as high as $\sim 300 \mathrm{~km} \mathrm{~s}^{-1}$, and steadily dropping down to $\sim 30 \mathrm{~km} \mathrm{~s}^{-1}$ for $\log g \sim 0$, as stars climb up the red giant branch (RGB). Red clump stars show a distribution of $\Delta \mathrm{RV}_{\max }$ values comparable to that of stars at the tip of the RGB, implying they have similar multiplicity characteristics. The observed attrition of high $\Delta \mathrm{RV}_{\max }$ systems in the RGB is consistent with a lognormal period distribution in the MS and a multiplicity fraction of 0.35 , which is truncated at an increasing period as stars become physically larger and undergo mass transfer after Roche Lobe overflow during $\mathrm{H}$-shell burning. The $\Delta \mathrm{RV}_{\max }$ distributions also show that the multiplicity characteristics of field stars are metallicitydependent, with metal-poor $([\mathrm{Fe} / \mathrm{H}] \lesssim-0.5)$ stars having a multiplicity fraction a factor of $2-3$ higher than metal-rich $([\mathrm{Fe} / \mathrm{H}] \gtrsim 0.0)$ stars. This has profound implications for the formation rates of interacting binaries observed by astronomical transient surveys and gravitational wave detectors, as well as the habitability of circumbinary planets.
\end{abstract}

Key words: binaries (including multiple): close - binaries: spectroscopic - stars: evolution - stars: fundamental parameters

\section{Introduction}

Stellar multiplicity plays a fundamental role in astrophysics. Interacting binary stars are the progenitors of all Type Ia $\mathrm{SNe}$ (Maoz et al. 2014), many core-collapse SNe (Sana et al. 2012), and a host of other astronomical sources, from high- and lowmass X-ray binaries to novae, cataclysmic variables, AM CVn stars, and most stellar sources of gravitational waves. Yet, our knowledge of the fundamental statistics of stellar multiplicity (multiplicity fraction, period distribution, mass ratio distribution, and eccentricity distribution) is still rudimentary, especially after the main sequence (MS) and beyond the solar

\footnotetext{
21 Einstein Fellow.

22 Alfred P. Sloan Fellow.
}

neighborhood (see Duchêne \& Kraus 2013; Moe \& Di Stefano 2017, for recent reviews). Solar-type MS stars closer than 25 pc have a roughly lognormal period distribution, with $\overline{\log P} \sim 5.0$ and $\sigma_{\log P} \sim 2.3$ for $P$ in days (Raghavan et al. 2010; see also our Figure 1), and a multiplicity fraction of $0.50 \pm 0.04$ for $\log P \leqslant 8$ after completeness corrections (Moe \& Di Stefano 2017). ${ }^{23}$ The multiplicity fraction on the MS is a strong function of primary mass, with more massive primaries having a higher likelihood of being in a multiple system (Lada 2006;

\footnotetext{
$\overline{23}$ Although it remains the best studied and most comprehensive census of multiple systems in the solar neighborhood, the Raghavan et al. (2010) sample is known to be incomplete for faint companions and very-low-mass ratios, especially at wide separations; see Chini et al. (2014) and Moe \& Di Stefano (2017) for discussions.
} 


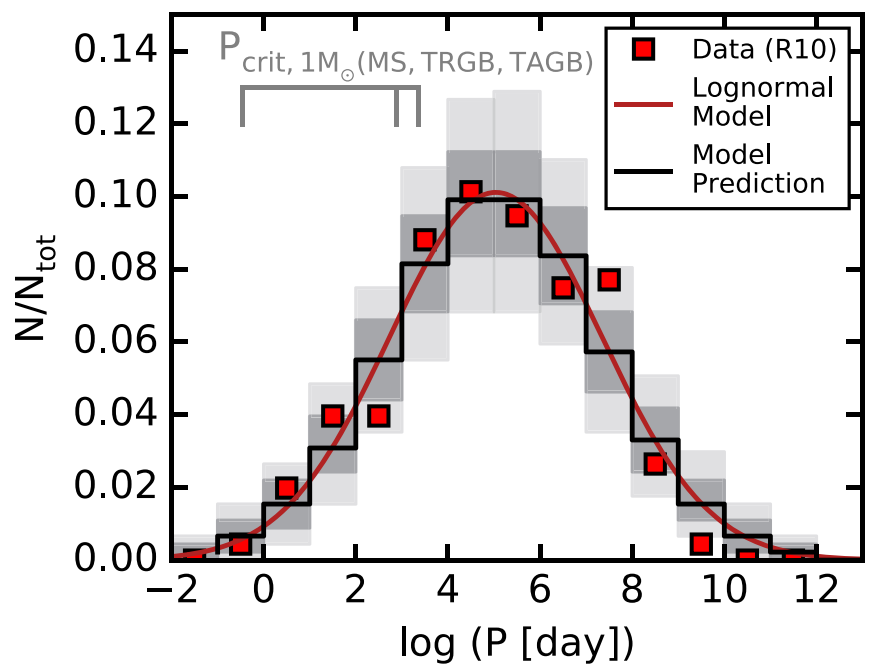

Figure 1. Period distribution for the Sun-like MS stars in Raghavan et al. (2010; red squares), together with a model $\operatorname{lognormal}$ distribution $(\overline{\log P}=5.0$ and $\sigma_{\log P}=2.3$, dark red solid line), and the predicted nominal (black), and $1 \sigma$ and $2 \sigma$ ranges (dark and light gray) from Poisson realizations of the model. The ruler in the top left corner indicates the critical period for Roche Lobe overflow (RLOF) in Sun-like models during the MS, at the tip of the red giant branch (RGB), and at the tip of the asymptotic giant branch (AGB).

Duchêne \& Kraus 2013), especially at short periods (Moe \& Di Stefano 2017), but the lognormal shape of the period distribution is robust below $\log P \sim 4$ for Sun-like stars (Latham et al. 2002; Melo 2003; Carney et al. 2005; Geller \& Mathieu 2012; Leiner et al. 2015; Moe \& Di Stefano 2017). The mass ratio has a roughly flat distribution, but Moe \& Di Stefano (2017) showed that this is not independent of the period distribution. The eccentricities of MS binaries follow a Maxwellian "thermal" distribution at intermediate periods, but tidal interactions circularize the orbits below $\log P \sim 1.1$ (Zahn 1989; Raghavan et al. 2010; Moe \& Di Stefano 2017).

Most observational studies of stellar multiplicity have focused on small samples of a few hundred objects in specific environments like the solar neighborhood or individual stellar clusters (e.g., Duquennoy \& Mayor 1991; Carney et al. 2003; Geller et al. 2008; Raghavan et al. 2010; Matijevic et al. 2011; Sana et al. 2012; Merle et al. 2017). In this context, intrinsic variations of the multiplicity statistics with parameters like effective gravity, age, metallicity, or disk/halo membership cannot be explored without addressing large observational biases. These intrinsic variations, if present, might affect the interplay between stellar multiplicity and stellar evolution, and impact the formation rates of interacting binaries (Gosnell et al. 2015; Andrews et al. 2016). There is a pressing need for multiplicity surveys with enough scope and precision to deal with these issues and effectively probe stellar multiplicity after the MS and across the Milky Way.

The Apache Point Observatory Galactic Evolution Experiment (APOGEE Majewski et al. 2017) within the Sloan Digital Sky Survey (SDSS; Gunn et al. 2006; Blanton et al. 2017), with its high-resolution, high-efficiency, multiplexed infrared spectrograph, has produced the first truly panoramic view of the stellar content of our Galaxy. APOGEE has targeted more than 150,000 stars at distances of up to $\sim 30 \mathrm{kpc}$, probing heavily obscured parts of the Galactic Disk. The APOGEE Stellar Parameter and Chemical Abundances Pipeline (ASPCAP, García Pérez et al. 2016) provides reliable measurements of effective temperatures ( $T_{\text {eff }}$ ), surface gravities $(\log g$ ), and chemical abundances for each of these targets, as well as highly precise radial velocities (RVs; Nidever et al. 2015). APOGEE also has a temporal dimension, with multiple spectra taken for each target, that enables studies of stellar multiplicity. In Troup et al. (2016), this temporal dimension was explored for a few thousand stars with seven or more RV measurements, which allowed them to derive orbital solutions and investigate the properties of stellar and substellar companions in detail. Here, we focus on the majority of APOGEE targets, which have only sparsely sampled RV curves.

For these stars, detailed orbital parameters cannot be measured (see Price-Whelan et al. 2017), but a statistical analysis of their RVs can still yield valuable insights on stellar multiplicity, its dependence on fundamental stellar properties, and its interplay with stellar evolution. Our work continues the statistical study of stellar multiplicity with sparsely sampled RV curves in SDSS that began with the study of white dwarfs (Badenes \& Maoz 2012; Maoz et al. 2012) and MS stars (Hettinger et al. 2015) drawn from the low-resolution optical spectra in the Sloan Extension for Galactic Understanding and Exploration survey (SEGUE; Yanny et al. 2009). Here, we explore the possibilities for these kind of studies that are opened up by the higher resolution of the infrared APOGEE spectrographs. Our work is organized as follows. In Section 2 we describe our sample selection. In Section 3 we examine the statistical properties of the RV variability in APOGEE stars, its theoretical interpretation, and the dependence with $\log g$ and metallicity. In Sections 4 and 5 we discuss our results and present our conclusions.

\section{Sample Selection and RV Measurements}

The DR13 APOGEE allstar file contains measurements for 163,278 targets (Albareti et al. 2017). From this sample, we removed all stars flagged as bad (STAR_BAD set in the ASPCAP flag bitmask, Holtzman et al. 2015) and stars targeted as telluric calibrators (bit 9 set in the apogee_target 2 mask; Zasowski et al. 2013). To restrict our measurements to the field, we also removed stars targeted as cluster members (bit 9 in the apogee_target 1 mask and bit 10 in the apogee_target 2 mask). To further ensure a well-characterized sample, we removed stars that did not have acceptable uncalibrated values of $T_{\text {eff }}$ and $\log g$ from ASPCAP. We did not use the values calibrated with asteroseismology because these are only available for giant stars, and we are interested in all the stellar evolution phases sampled by APOGEE. This calibration shifts the surface gravities for giants by $\sim-0.2$ dex, which is not critical for our goals. This left 122141 entries, for which we examined the individual RVs in the allVisit file. We used only visit spectra that were deemed of high enough quality to contribute to the combined APOGEE spectrum (the VISIT_PK indices, Holtzman et al. 2015; Nidever et al. 2015), and we further required a $\mathrm{S} / \mathrm{N}$ of at least 40 in each of the visit spectra. A total of 91,246 unique stars in APOGEE have two or more RVs that pass these quality cuts.

The stars in our main sample are placed in an observational Hertzsprung-Russell (HR) diagram in Figure 2. For context, we have overlaid stellar models from the MESA Isochrone and Stellar Tracks collaboration (MIST; Paxton et al. 2011, 2013, 2015; Choi et al. 2016), spanning the phases between core $\mathrm{H}$ ignition and core He exhaustion (beginning of the MS to the end of the horizontal branch) for zero-age main sequence masses 


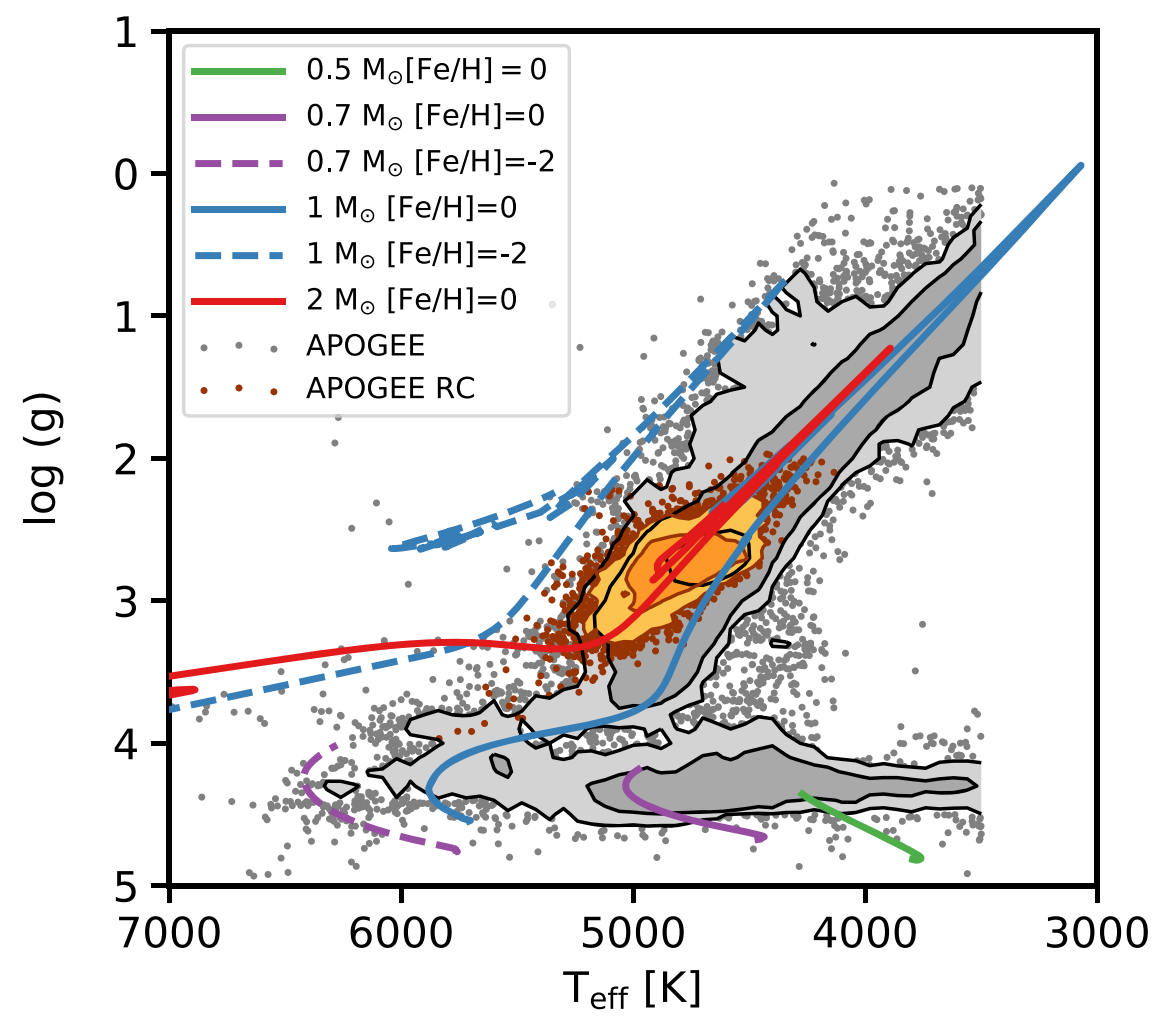

Figure 2. Observational HR diagram for the APOGEE targets in our main sample (gray dots and density contours), including the RC stars from Bovy et al. (2014; orange dots and density contours). Selected MIST models from Choi et al. (2016) are overlaid for context.

between 0.5 and $2 M_{\odot}$. The sample is dominated by red giant branch (RGB) stars at several stages of their evolution, from the subgiant branch to almost the tip of the RGB $(3.75 \gtrsim \log g \gtrsim 0)$. There are also substantial contributions from MS stars and subgiants ( $\log g \gtrsim 3.75$ and $T_{\text {eff }}$ between 3500 and $6500 \mathrm{~K}$ ), as well as red clump (RC) stars, which can be identified by their location in color-metallicity-log $g-T_{\text {eff }}$ space (Bovy et al. 2014). The 15667 RC stars from the DR13 version of the Bovy et al. (2014) catalog that pass our quality cuts are shown separately in Figure 2. Our main sample probably contains some AGB stars, which overlap the RGB in $\log g, T_{\text {eff }}$ space, but given the short lifetimes of all phases of stellar evolution after core $\mathrm{He}$ exhaustion, this contamination should be small and we ignored it in our analysis.

The properties of the stars in our main sample are typical of APOGEE targets with high-quality spectra. The metallicities range from $[\mathrm{Fe} / \mathrm{H}]=0.5$ to -2.5 (the range of models available in ASPCAP), with the bulk of the targets lying between 0.0 and -0.5 (see Figure 3). Ness et al. (2016) derived ages and masses for some of the RGB and RC stars in APOGEE by applying the label transfer tool The Cannon (Ness et al. 2015) to a training set of stars with asteroseismic masses from APOKASC (Pinsonneault et al. 2014). This study probably yields the most accurate measurements for the masses and ages of APOGEE targets, but it does not extend to $\log g$ values above 3.29 or below 1.36. In Figure 4 we show the mass histograms for the RGB stars with the highest and lowest $\log g$ values in the study by Ness et al. (2016) — the RC mass distribution is omitted for clarity, but it is similar to that at the highest $\log g$ range. From these distributions, we conclude that most RGB and RC stars in APOGEE have masses close to $1 M_{\odot}$, with few below that value or above $2 M_{\odot}$. The mass

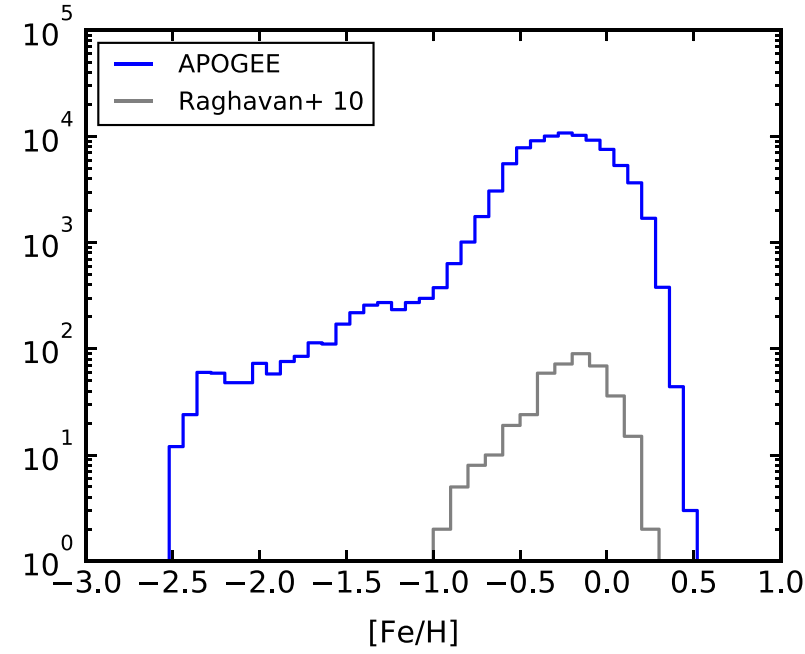

Figure 3. Distribution of $[\mathrm{Fe} / \mathrm{H}]$ values in the APOGEE targets that pass our quality cuts (blue histogram), compared to the sample from Raghavan et al. (2010; gray histogram).

distribution in the MS is not as well-characterized, but it must be skewed toward lower masses (see Figure 2).

The APOGEE data reduction pipeline (Nidever et al. 2015) and the ASPCAP pipeline (García Pérez et al. 2016) operate on the assumption that each spectrum is dominated by flux emitted by a single stellar photosphere, but this is not necessarily true. Some stars will have companions of comparable brightness, such that the combined spectrum will reveal spectral lines from both components, often offset in wavelength according to their relative line-of-sight velocities. These so-called double-lined spectroscopic binaries, or SB2s, can confound the APOGEE 


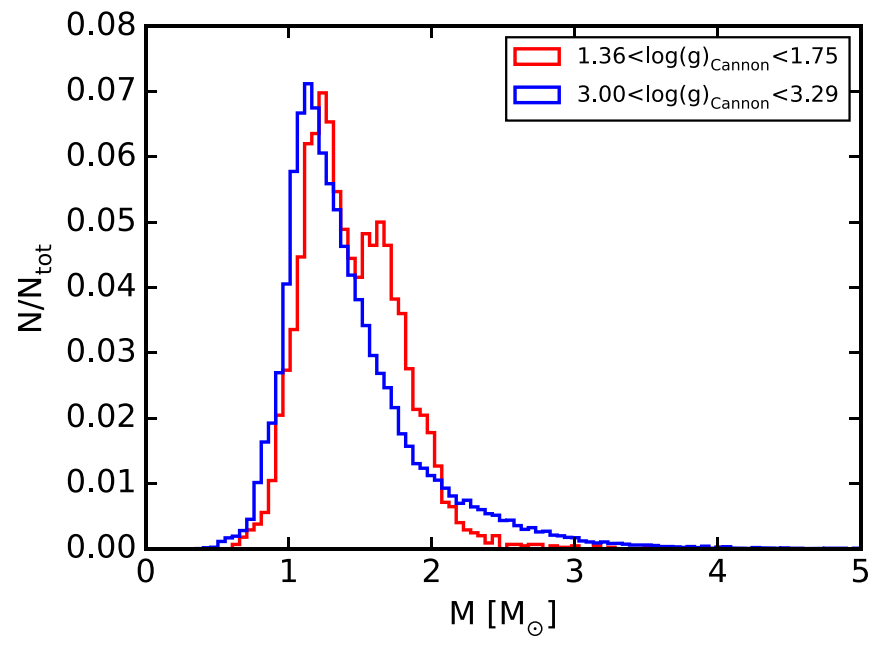

Figure 4. Mass distribution of APOGEE targets classified as RGB stars in two representative $\log g$ ranges from the re-analysis of the spectra by Ness et al. (2016).

and ASPCAP pipelines, potentially introducing substantial errors into the stellar parameters (e.g., $T_{\text {eff }}, \log g,[\mathrm{Fe} / \mathrm{H}]$, etc.; see El-Badry et al. 2018a) or RVs that are inferred from the spectrum. To account for these effects, we drew from a preliminary catalog of $1200 \mathrm{SB} 2 \mathrm{~s}$ in the DR13 data set, constructed using techniques developed to identify and characterize SB2s in the APOGEE/IN-SYNC survey of local star-forming regions (Fernandez et al. 2017). Only 482 of these SB2 stars pass the quality cuts used to construct our sample, most of them with $\log g$ values in the MS and subgiant region. For these targets, we replace the RVs calculated by the APOGEE pipeline with the RV extracted from the highest peak in the cross-correlation function at each epoch, which provides a more reliable measure of the RV of the photometric primary. A similar study using a data-driven spectral fitting method for the MS was recently published by El-Badry et al. (2018b).

\section{3. $\Delta R V_{\max }$ : A Figure of Merit for Sparsely Sampled RV Curves}

\section{1. $\Delta R V_{\max }$ and $\log g$}

Among the targets in our sample, most (62\%) have three RVs that pass quality cuts, $22 \%$ have only two RVs, and $16 \%$ have four or more. A simple figure of merit to characterize these sparsely sampled RV curves is the difference between the highest and lowest measured $R V$ s for each star, $\Delta \mathrm{RV}_{\max }=$ $\max \left(\mathrm{RV}_{n}\right)-\min \left(\mathrm{RV}_{n}\right)$ (see Badenes \& Maoz 2012 and Maoz et al. 2012, for discussions). In Figure 5 we show the values of $\Delta R V_{\text {max }}$ as a function of $\log g$ for the targets in our main sample. The maximum value of $\Delta \mathrm{RV}_{\max }$ measured by APOGEE is a strong function of $\log g$, with MS stars showing values as high as $\sim 300 \mathrm{~km} \mathrm{~s}^{-1}$, and stars near the tip of the RGB only going to $\sim 30 \mathrm{~km} \mathrm{~s}^{-1}$.

This trend in $\Delta \mathrm{RV}_{\text {max }}$ versus log $g$ can be best appreciated in the cumulative histograms shown in Figure 6, which highlight the tail of the $\Delta \mathrm{RV}_{\max }$ distribution above a few $\mathrm{km} \mathrm{s}^{-1}$. Here, we have divided our main sample of non-RC stars into eight $\log g$ bins, one for subgiant and MS stars $(\log g>3.25)$, and seven for RGB stars down to $\log g \sim 0$. These bins have roughly the same number of stars $(\sim 8000)$, except for the MS/subgiant bin, which has $\sim 19,000$ (see Table 1). The $\mathrm{MS} /$ subgiant bin could in principle be resolved into smaller bins, but given the known limitations of the ASPCAP pipeline for high $\log g$ stars, we felt this was not justified. The histograms in Figure 6 show that there is a clear attrition of high $\Delta \mathrm{RV}_{\max }$ values as stars climb the $\mathrm{RGB}$, and shows that the distribution of $\Delta R V_{\text {max }}$ in the RC closely resembles that near the tip of the RGB.

\subsection{From Periods and Eccentricities to $\Delta R V_{\max }$}

The highest value of $\Delta \mathrm{RV}_{\max }$ in a large sample of stars will correspond to the edge-on $\left(i=90^{\circ}\right)$ binary systems with the shortest possible orbital periods. This should be the critical period for RLOF,

$$
P_{\text {crit }}=2 \pi \mathcal{R}(q) \sqrt{\frac{R^{3}}{G M}},
$$

where $\mathcal{R}(q)$ is the ratio between the radius of the Roche Lobe and the orbital separation (which can be approximated by 0.38 for $q=1$, Eggleton 1983), and $M$ and $R$ are the mass and radius of the photometric primary (i.e., the star that contributes most of the flux in the APOGEE bands). For any period $P$, the peak-to-peak amplitude of the RV curve of an edge-on orbit, $\Delta \mathrm{RV}_{\mathrm{pp}}$, is twice the semi-amplitude $K$,

$$
\Delta \mathrm{RV}_{\mathrm{pp}}=2 K=\frac{2}{\sqrt{1-e^{2}}}\left(\frac{\pi G M}{2 P}\right)^{1 / 3},
$$

where $e$ is the eccentricity. It follows from Equations (1) and (2) that the value of $\Delta \mathrm{RV}_{\mathrm{pp}}$ at $P_{\text {crit }}$ for a circular orbit with $q=1$ is uniquely determined by the mass and radius of the primary. For stars whose effective gravity $g$ is measured directly, which applies to our APOGEE targets, these equations can be combined into a simple expression,

$$
\Delta \mathrm{RV}_{\mathrm{pp}, P_{\text {crit }}}=0.87(G M g)^{1 / 4} \text {. }
$$

Therefore, a theoretical maximum for $\Delta \mathrm{RV}_{\mathrm{pp}}$ can be estimated for any measured $\log g$ just by assuming a mass, as shown by the solid lines overlaid on Figure 5. For $M=1 M_{\odot}$, $P_{\text {crit }}$ is 0.35 days in the MS $(\log g \sim 4.5)$ and 2.1 years at the tip of the RGB $(\log g \sim 0)$, and $\Delta \mathrm{RV}_{\mathrm{pp}}$ should vary between 400 and $30 \mathrm{~km} \mathrm{~s}^{-1}$, as shown schematically in Figure 7. These theoretical maxima compare well with the $\Delta \mathrm{RV}_{\max }$ measurements in Figures 5 and 6, allowing for the fact that the observed systems have a distribution of primary masses, mass ratios, and inclinations, and their RV curves are sparsely sampled by APOGEE.

Orbital eccentricities will also affect $\Delta \mathrm{RV}_{\max }$, even though tidal interactions will circularize the orbits with the shortest periods, i.e., those contributing the largest $\Delta R V_{\text {max }}$ at any given $\log g$, on timescales that are comparable to the evolutionary timescale on the RGB (Meibom \& Mathieu 2005). For Sun-like MS stars, Raghavan et al. (2010) measured a circularization period of $P_{\text {circ }} \sim 12$ days $\left(\log P_{\text {circ }} \sim 1.1\right)$. Unfortunately, there is no comprehensive observational study of $P_{\text {circ }}$ in RGB stars with varying $\log g$. From the theory of tides, Verbunt $\&$ Phinney (1995) derived the value of $P_{\text {circ }}$ as a function of the depth of the convective envelope in the RGB. Taking the depth of the convective envelope from the $1 M_{\odot}$ model of solar metallicity by Choi et al. (2016), the expressions in Verbunt \& Phinney (1995) predict a steady increase of $P_{\text {circ }}$ with decreasing $\log g$, with $\log P_{\text {circ }} \sim 4.2$ at the tip of the RGB (Figure 8). This provides a baseline theoretical scenario for 


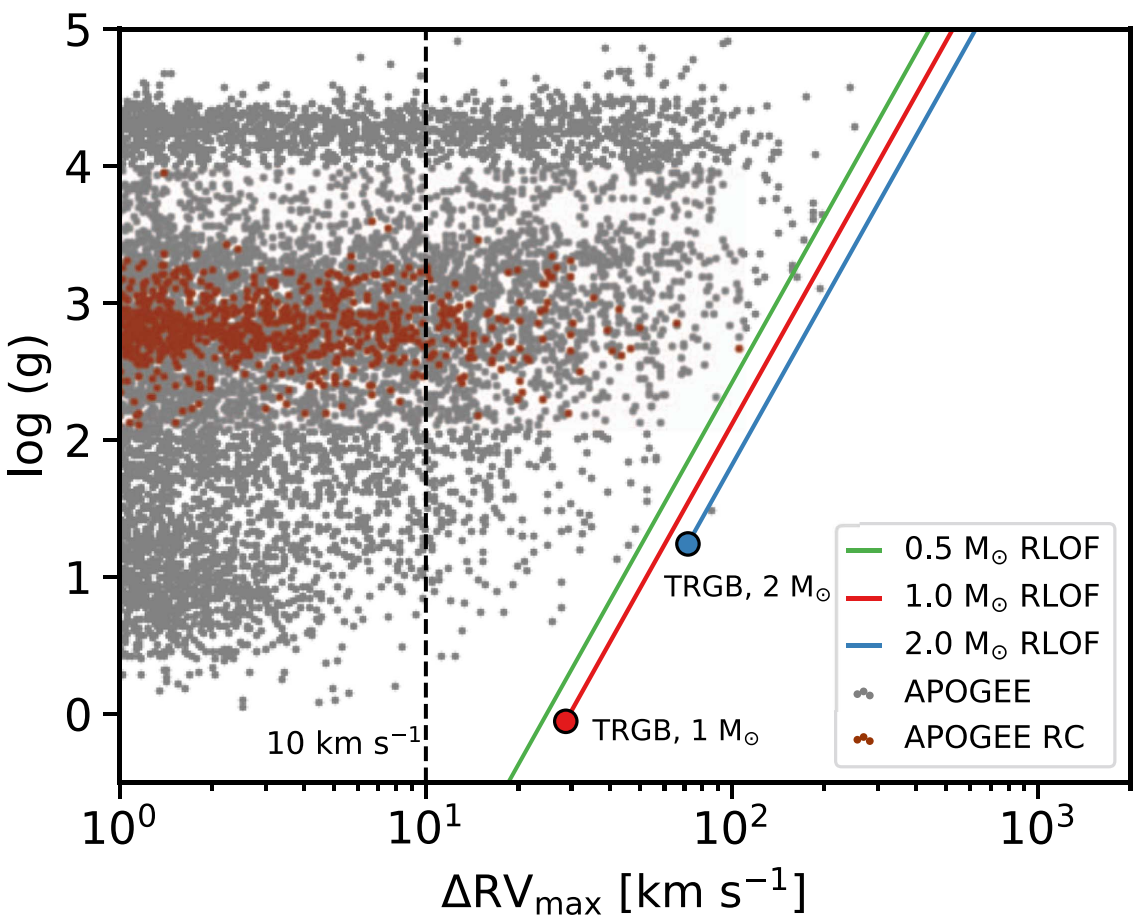

Figure 5. Distribution of $\Delta \mathrm{RV}_{\max }$ values for APOGEE stars in our main sample (gray dots) and RC sample (dark red dots) as a function of log $g$. The solid lines indicate the maximum value of $\Delta \mathrm{RV}_{\max }$ (for $q=1$ and $i=90^{\circ}$ ) at the critical period for RLOF in stars of 0.5 (green), 1 (red), and 2 (blue) $M_{\odot}$ as a function of log $g$. The position of the tip of the RGB (the lowest value of $\log g$ ) in MIST models of solar metallicity is indicated by the terminal symbols for 1 and $2 M_{\odot}$ stars.

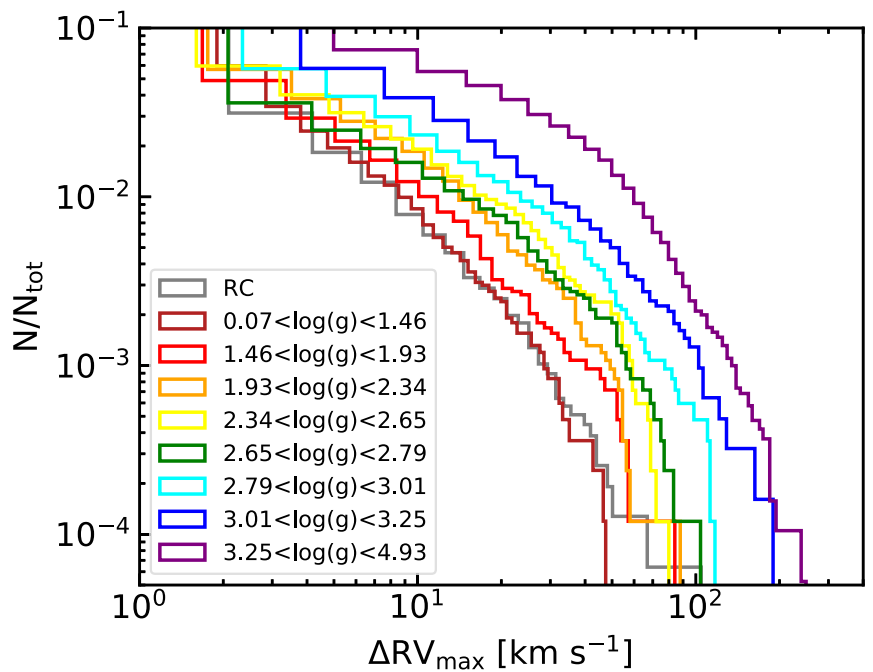

Figure 6. Cumulative histograms of $\Delta \mathrm{RV}_{\max }$ for eight $\log g$ bins in the main sample, covering the range between the MS and the tip of the RGB (colored plots), plus the RC catalog (gray plot).

orbital circularization in the RGB. The maximum eccentricity allowed at any period above $P_{\text {circ }}$ is limited by angular momentum conservation, and can be approximated as

$$
e_{\max }=\sqrt{1.0-\left(\frac{P_{\text {circ }}}{P}\right)^{2 / 3}}
$$

(Mazeh 2008), which has been shown to be in good agreement with Kepler observations of "heartbeat stars" (Shporer et al. 2016). Note that the period exponents in Equations (2) and (4) cancel out, therefore the maximum $\Delta \mathrm{RV}_{\mathrm{pp}}$ remains constant for periods above $P_{\text {circ }}$ (horizontal blue and red lines in Figure 7). In practice, however, $\Delta \mathrm{RV}_{\max }$ values close to this theoretical upper limit for highly eccentric systems are hard to observe at periods much longer than $P_{\text {circ }}$ because (1) there is a limit to the temporal baseline that can be probed in any RV survey, (2) systems with $e \sim e_{\max }$ are rare, and (3) at high eccentricities it becomes increasingly difficult to capture the full dynamic range of RV with a sparse sampling, since most of the variation happens in a brief time interval close to periastron. In any case, orbital eccentricities will break down the simple relationship between $P$ and $\Delta \mathrm{RV}_{\mathrm{pp}}$ shown in Equation (2) for periods longer than $P_{\text {circ }}$.

To help visualize the effects of the APOGEE sampling on the distribution of $\Delta R V_{\max }$, we have added an inset to Figure 7 that shows the fraction of stars that could in principle probe the full value of $\Delta R V_{p p}$, because they have temporal baselines longer than half a given period. The temporal baselines of individual APOGEE targets range between 0.8 and 1043 days, with a median of 40 days. The fraction of targets that can fully sample the maximum $\mathrm{RV}$ range as a function of period falls rapidly above $\log P \sim 1.7$, but it remains above $10 \%$ at $\log P \sim 2.8$, which is the value of $P_{\text {crit }}$ at the tip of the RGB for systems with $1 M_{\odot}$ primaries. There are no large systematic variations of the distribution of temporal baselines or the number of visits with either $\log g$ or metallicity in our APOGEE targets.

\subsection{Measurement Errors and Multiple Systems}

Even though the APOGEE data reduction pipeline reports RV errors below $0.1 \mathrm{~km} \mathrm{~s}^{-1}$ (Nidever et al. 2015), Cottaar et al. (2014) found evidence that these errors might be underestimated by as much as a factor $\sim 3$. The $\Delta \mathrm{RV}_{\max }$ distributions measured by APOGEE also indicate that either the average RV errors are larger than reported by the pipeline or there is an additional source of scatter in the individual RV measurements. 
Table 1

Systems with $\Delta \mathrm{RV}_{\max }>10 \mathrm{~km} \mathrm{~s}^{-1}$

\begin{tabular}{|c|c|c|c|c|c|}
\hline Sample & $\begin{array}{c}\log g \\
\text { Range }\end{array}$ & $\begin{array}{c}\text { Median } \\
\log g\end{array}$ & $N$ & $N_{\Delta \mathrm{RV}_{\max }>10 \mathrm{~km} \mathrm{~s}^{-1}}$ & $N_{\Delta \mathrm{RV}_{\max >10 \mathrm{~km} \mathrm{~s}^{-1}}} / N$ \\
\hline MS/Subg. & $3.250-4.932$ & 4.203 & 19045 & 1051 & 0.0552 \\
\hline RGB 6 & $3.009-3.250$ & 3.133 & 6211 & 200 & 0.0322 \\
\hline RGB 4 & $2.649-2.791$ & 2.722 & 8385 & 114 & 0.0136 \\
\hline RGB 3 & 2.339-2.649 & 2.524 & 8412 & 158 & 0.0188 \\
\hline RGB 2 & $1.931-2.339$ & 2.141 & 8393 & 135 & 0.0161 \\
\hline $\mathrm{RC}$ & $2.250-3.250$ & 2.766 & 15667 & 100 & 0.0064 \\
\hline
\end{tabular}

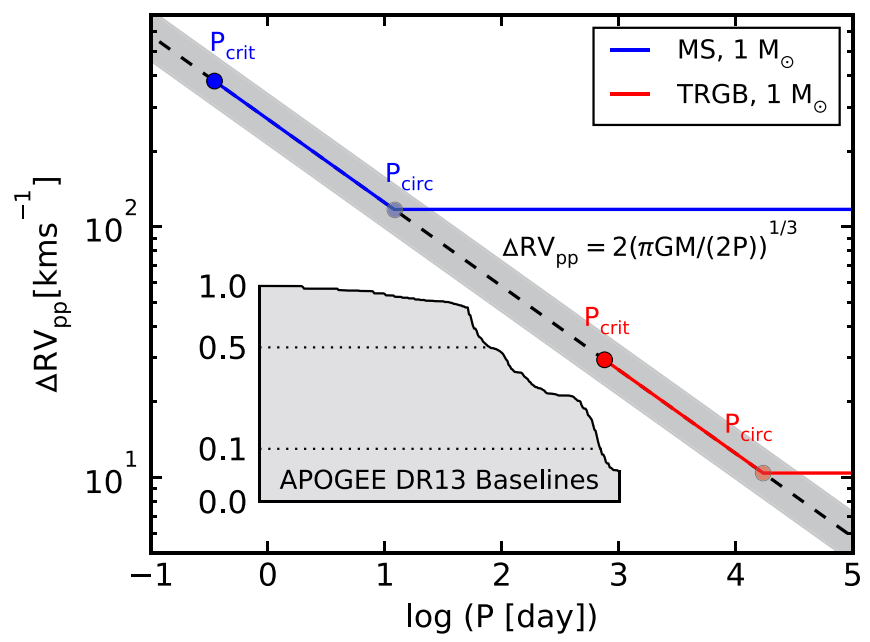

Figure 7. Relationship between $P$ and $\Delta \mathrm{RV}_{\mathrm{pp}}$ for edge-on, $q=1$ systems with circular orbits and $1 M_{\odot}$ primaries (dashed black line). The range of $\Delta \mathrm{RV}_{\mathrm{pp}}$ for systems with primaries between 0.5 and $2 M_{\odot}$ is shown by the gray band. The maximum value of $\Delta \mathrm{RV}$ pp for eccentric systems with $1 M_{\odot}$ primaries is shown by the solid colored lines: blue for the MS and red for the tip of the RGB $(\log g=0)$, with the values of $P_{\text {crit }}$ (from Equation (1)) and $P_{\text {circ }}$ (from observations and the theory of Verbunt \& Phinney 1995 applied to the MIST models of Choi et al. 2016) marked by symbols. The inset shows the fraction of APOGEE targets in our sample that have temporal baselines larger than half a given period, and therefore could theoretically probe the full range of the RV curve.

In a survey with a large number of sparsely sampled RV curves, the $\Delta \mathrm{RV}_{\max }$ distribution usually has a core of low values dominated by measurement errors and a tail of high values from bona fide RV variables (Maoz et al. 2012). Even without a detailed understanding of the RV errors, the transition between tail and core can often be determined empirically, and objects above the transition can be identified as bona fide multiple systems (see Badenes \& Maoz 2012; Maoz \& Hallakoun 2016). In the left panel of Figure 9 we show the $\Delta \mathrm{RV}_{\max }$ distribution in our APOGEE MS/Subgiant sample, as well as that of the stars in the highest and lowest $[\mathrm{Fe} / \mathrm{H}]$ terciles within this sample. The core/tail transition can be clearly seen at $\sim 0.7 \mathrm{~km} \mathrm{~s}^{-1}$, with the high and lowmetallicity terciles having somewhat lower and larger transition values, respectively. Also shown in Figure 9 are models where a Gaussian distribution of RV errors is sampled with the APOGEE visits. A comparison to these models indicates a value of $\sigma_{\text {RVerr }}$, which is close to $0.2 \mathrm{~km} \mathrm{~s}^{-1}$ for the MS/Subgiant sample. These $\Delta \mathrm{RV}_{\max }$ distributions, and the inferred RV errors, are representative of the majority of our $\log g$ samples. The one exception is shown in the right panel of

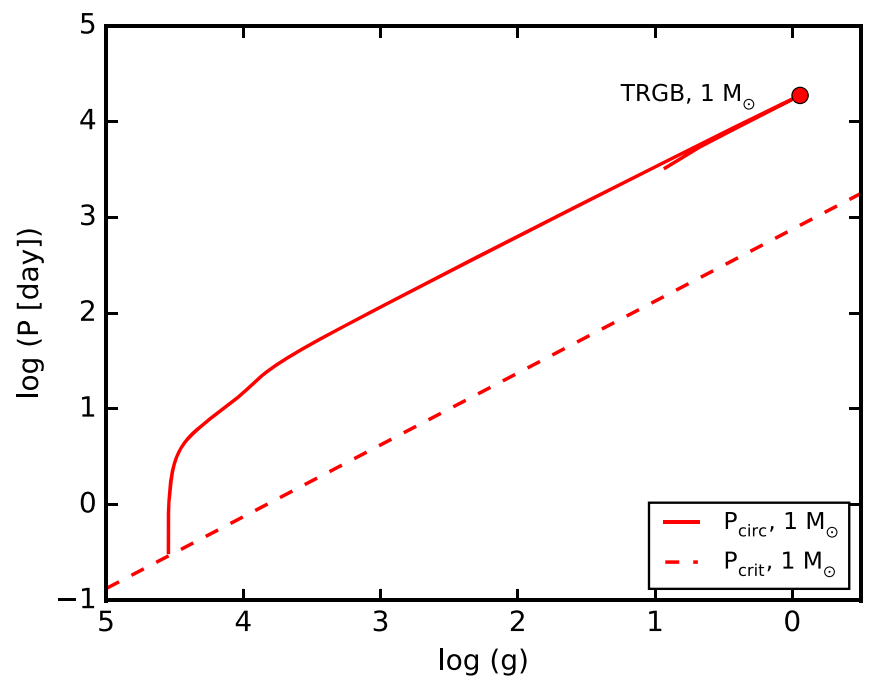

Figure 8. Values of $P_{\text {crit }}$ and $P_{\text {circ }}$ as a function of $\log g$ for $1 M_{\odot}$ stars. The values for $P_{\text {crit }}$ are calculated from Equation (1). The values for $P_{\text {circ }}$ are calculated using the theory of Verbunt \& Phinney (1995), with the depth of the convective envelope taken from the $1 M_{\odot},[\mathrm{Fe} / \mathrm{H}]=0$ model from Choi et al. (2016).

Figure 9. The APOGEE targets with the lowest $\log g$ values $(0.07>\log g>1.46)$ have noticeably broader $\Delta \mathrm{RV}_{\max }$ cores, with a core/tail transition as high as $\sim 5.0 \mathrm{~km} \mathrm{~s}^{-1}$ for the stars in the lowest $[\mathrm{Fe} / \mathrm{H}]$ tercile. Some of these stars might have RV errors as high as $\sigma_{\mathrm{RVerr}} \sim 1 \mathrm{~km} \mathrm{~s}^{-1}$.

This behavior of the RV errors is qualitatively consistent with the properties of the cross-correlation procedure used by the APOGEE data reduction pipeline to measure RVs, which is sensitive to parameters that affect the number, depth, and broadness of spectral lines, like $\log g$ and $[\mathrm{Fe} / \mathrm{H}]$. Quantitatively, the extent of our $\Delta \mathrm{RV}_{\max }$ cores seems to require larger $\mathrm{RV}$ errors than those reported by the pipeline (see Section 8.3 in Nidever et al. 2015). This might be due to a systematic underestimation of the RV errors by the pipeline or to the presence of a non-Gaussian tail of RV errors, but a more likely explanation is RV jitter, which can introduce a scatter of up to a few $\mathrm{km} \mathrm{s}^{-1}$ in the spectra of RGB stars, especially at low $\log g$ (Carney et al. 2003; Hekker et al. 2008). To ensure that RV errors, whatever their origin, will not affect our analysis, and that we will be able to compare stars of different $\log g$ and $[\mathrm{Fe} / \mathrm{H}]$ without biases, we define a conservative threshold of $\Delta \mathrm{RV}_{\text {max }}=10 \mathrm{~km} \mathrm{~s}^{-1}$ to identify a system as a bona fide multiple. This value is well within the tail of the $\Delta R V_{\max }$ distribution even in the most unfavorable cases shown in 

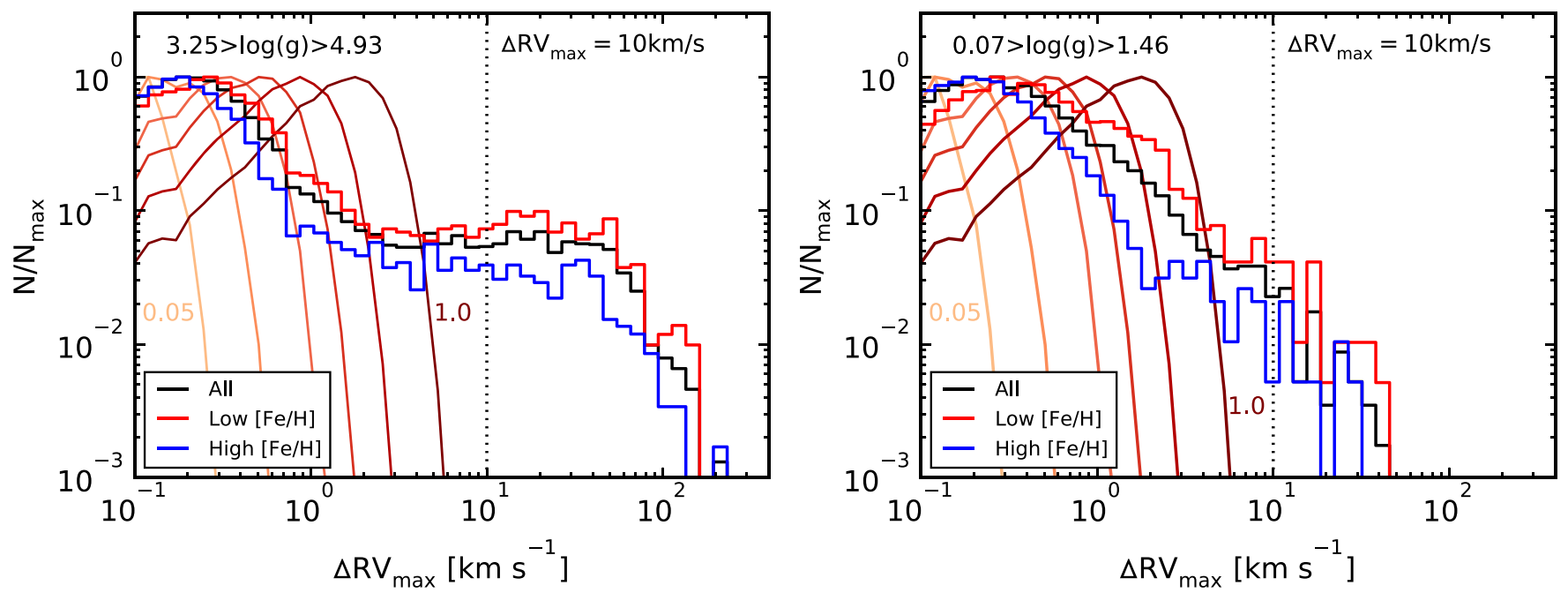

Figure 9. Logarithmic $\Delta \mathrm{RV}_{\max }$ distributions for the highest (left) and lowest (right) log $g$ subsamples. For each subsample, we show all stars with the black histogram, as well as the top tercile in $[\mathrm{Fe} / \mathrm{H}]$ (blue histogram) and the bottom tercile in $[\mathrm{Fe} / \mathrm{H}]$ (red histogram). The orange-to-red plots represent simulations of $\Delta \mathrm{RV}_{\text {max }}$ measurements without binaries, using the APOGEE epochs to sample constant $\mathrm{RV}$ curves with Gaussian $\mathrm{RV}$ errors with $\sigma_{\mathrm{RV} \text { err }}=0.05$ (lightest shade), 0.1 , $0.2,0.3,0.5$, and 1.0 (darkest shade) $\mathrm{km} \mathrm{s}^{-1}$.

Figure 9, but is low enough to yield a total of 2088 bona fide multiple systems, with enough systems in each $\log g$ sample for statistical analysis (see Table 1). From Equation (2), the minimum period required to get $\Delta \mathrm{RV}_{\max }=10 \mathrm{~km} \mathrm{~s}^{-1}$ is $\log P=4.3$ ( $2 \times 10^{4}$ days, or 54 years $)$, which is close to the expected circularization period for Sun-like stars at the tip of the RGB (Figure 8). However, the temporal baselines in APOGEE cannot probe such long periods (see Figure 7). The longest period we are sensitive to with this $\Delta R V_{\max }$ threshold is that which can produce an RV shift of at least $10 \mathrm{~km} \mathrm{~s}^{-1}$ in the longest baselines available in APOGEE $\left(\sim 10^{3}\right.$ days $)$, which is about $\log P \sim 3.3$, or $5.5 \mathrm{yr}$.

\subsection{Physical Interpretation: Monte Carlo Models of $\Delta R V_{\max }$}

The relationship between $\Delta \mathrm{RV}_{\max }$ and $\log g$ shown in Figures 5 and 6 can be understood qualitatively through the equations introduced in Section 3.2 and the interplay between stellar multiplicity and stellar evolution. After $\sim 1 M_{\odot}$ primaries exhaust $\mathrm{H}$ in their cores and leave the MS, they climb the RGB and their $\log g$ drops from $\sim 4.5$ to $\sim 0$ as their radii increase from $\sim 1$ to $\sim 170 R_{\odot}$. For those in multiple systems, the maximum value of $\Delta \mathrm{RV}_{\max }$ allowed for $P=P_{\text {crit }}$ drops from $\sim 400$ to $\sim 30 \mathrm{~km} \mathrm{~s}^{-1}$ (Equation (3)). Because we cannot find any multiple systems in APOGEE with $\triangle \mathrm{RV}_{\max }$ values above these limits (i.e., to the right of the solid lines in Figure 5), all systems with $P<P_{\text {crit }}$ must have been removed from the sample by some efficient process. This removal of short $P$ systems also results in a lower number of stars observed at all values of $\Delta \mathrm{RV}_{\max }$, as seen in Figure 6, due to the projection effect of random orbital inclinations (multiply Equation (3) by a factor $\sin i$ that is randomly distributed between 0 and 1 ). After core He ignition, the stars settle on the $\mathrm{RC}$ and their radii decrease again to $\sim 10 R_{\odot}$, but their $\Delta \mathrm{RV}_{\max }$ distribution remains similar to that of the larger stars at the tip of the RGB, because their short-period companions have already been removed during shell $\mathrm{H}$ burning.

A detailed quantitative evaluation of this scenario, including constraints on multiplicity fractions and period distributions, would require forward modeling of the APOGEE $\Delta \mathrm{RV}_{\max }$ measurements within a hierarchical Bayesian scheme, taking into account all the relevant stellar properties and the details of the mass distributions and tidal interactions for the entire sample (e.g., see Maoz et al. 2012; Walker et al. 2015). We leave that analysis for future work, and here we examine the main physical implications of our observations using a simpler method.

We generate artificial populations of stars that can be sampled with the APOGEE epochs using a Monte Carlo code. Our code assumes that all photometric primaries are $1 M_{\odot}$ (see Figure 4 and accompanying discussion), that the distribution of mass ratios is flat (a good first approximation for short-period companions to Sun-like stars, Moe \& Di Stefano 2017), and that orbital inclinations are random (i.e., the distribution of $\cos i$ is uniform). For each run, we choose a MS multiplicity fraction $f_{m}$ and $^{24}$ an effective gravity $\log g$. We adopt the period distribution of Raghavan et al. (2010); see Figure 1 and accompanying discussion), which we truncate at the value of $P_{\text {crit }}$ that corresponds to the chosen $\log g$ (Equation (1)). We assume that all systems with $P<P_{\text {crit }}$ have lost their companions and can be considered single. We calculate $P_{\text {circ }}$ from the theory of Verbunt \& Phinney (1995) and the $1 M_{\odot}$, $[\mathrm{Fe} / \mathrm{H}]=0$ model of Choi et al. (2016) (Figure 8), and assume that all orbits with $P_{\text {crit }}<P \leqslant P_{\text {circ }}$ are circular. For longer periods, the eccentricity is drawn from a uniform distribution (Moe \& Di Stefano 2017) between 0 and $e_{\max }$ (Equation (4)). We generate $N$ systems with these parameters, each of which is sampled with the epochs (number of visits and time lags between visits) from a random APOGEE target, with the orbital phase of the first visit drawn from a uniform distribution between 0 and $2 \pi$. Thus, the Monte Carlo code captures the main physics affecting the values of $\Delta R V_{\max }$ with only three free parameters: $f_{m}, \log g$, and $N$.

In Figure 10 we compare our Monte Carlo simulations with the fraction of targets with $\Delta \mathrm{RV}_{\max } \geqslant 10 \mathrm{~km} \mathrm{~s}^{-1}$ observed by

\footnotetext{
${ }^{24}$ Although we only consider binary systems, we call this a multiplicity fraction for consistency. In practice, most hierarchical multiple systems contain a tight inner binary that is responsible for most of the RV variation (Tokovinin et al. 2006; Duchêne \& Kraus 2013).
} 


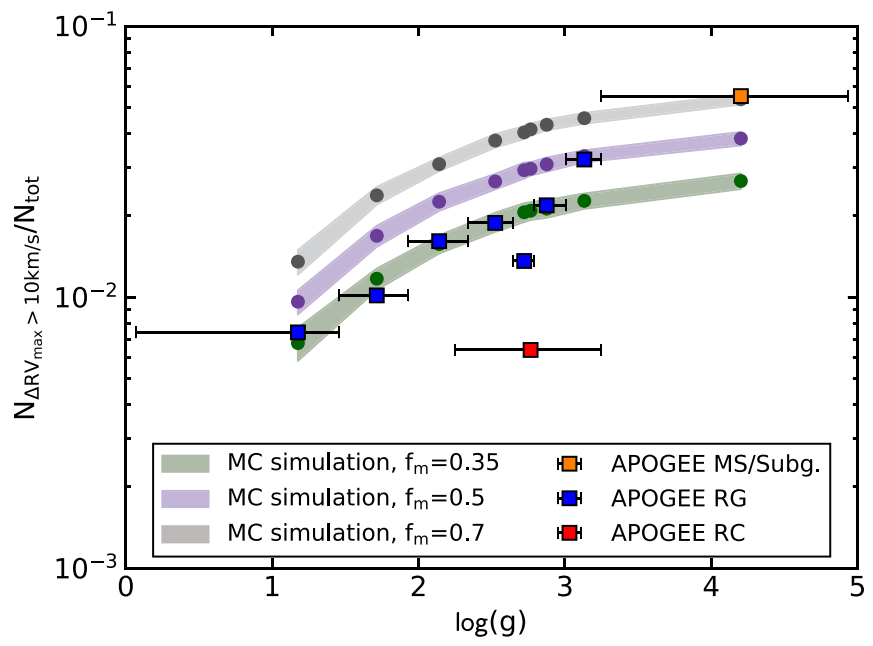

Figure 10. Fraction of systems with $\Delta \mathrm{RV}_{\max }>10 \mathrm{~km} \mathrm{~s}^{-1}$ in each $\log g$ subsample (orange square for the MS/subgiant sample, and blue squares for the RGB samples), as well as the RC catalog (red square). The symbols are placed at the median $\log g$ of each sample, with the error bars giving the range of $\log g$ values. The circles and colored bands represent the results of the Monte Carlo simulations described in Section 3.4, with $1 \sigma$ intervals calculated by running 100 instances of each simulation with $N=8300$.

APOGEE in each of the $\log g$ samples and in the RC catalog (Table 1). The attrition of high $\Delta \mathrm{RV}_{\text {max }}$ (short $P$ ) systems as stars climb the RGB is clearly seen in the APOGEE data, but the trend seems to break at $\log g \sim 2.7$ (sample RGB 4 in Table 1). This is the region of the HR diagram where the density peaks of RGB and RC stars overlap (see Figure 2), which suggests that the lower fraction of high $\Delta \mathrm{RV}_{\max }$ systems in this sample might be due to a contamination from unidentified $R C$ stars with lower $\Delta R V_{\max }$ values. This is not surprising, since the RC catalog of Bovy et al. (2014) is designed to be pure, rather than complete (see Price-Jones \& Bovy 2018).

Setting aside the issue of the RC contamination, our Monte Carlo simulations reproduce the observed behavior of $N_{\Delta \mathrm{RV}_{\max }} \geqslant 10 \mathrm{~km} \mathrm{~s}^{-1} / N$ as a function of $\log g$ quite well for RGB stars. The value observed in the RC is close to that predicted by our simulations at the tip of the RGB, as expected in the framework of our physical model. In the RGB, we obtain the best match to the observations for $f_{m}=0.35$. The RGB sample with the highest $\log g(\sim 3.1)$ and the MS/subgiant sample seem to require higher values of $f_{m}$, but these deviations should be interpreted with caution. The APSCAP pipeline was not designed for stars with high $\log g$, particularly at low $T_{\text {eff }}$, so the fitted stellar parameters in these samples might be subject to systematic uncertainties (García Pérez et al. 2016; note the mismatch between data and models in this region of Figure 2). Even taking the fitted stellar parameters at face value, it is clear from Figure 2 that these samples must have a primary mass distribution that is significantly different from the bulk of RGB and RC stars in APOGEE. Our Monte Carlo code assumes a single value of $f_{m}$ for all stars, which is not a valid approximation if there is a broad enough range of primary masses (Lada 2006; Moe \& Di Stefano 2017). Furthermore, the mass ratio distribution, MS period distribution, and tidal circularization model in our code are only appropriate for $1 M_{\odot}$ primaries. In view of this, the Monte Carlo simulations for the two highest $\log g$ samples might not reflect the underlying properties of the APOGEE targets. We will discuss our measurement of $f_{m}$ in more detail in Section 4.

\section{5. $\Delta R V_{\max }$ and Metallicity}

The relationship between stellar multiplicity and metallicity is a controversial topic. Some numerical simulations of star formation show that metallicity should have a strong effect on multiplicity, with lower-metallicity clouds showing higher fragmentation rates and smaller initial separations for binary systems (Machida 2008; Machida et al. 2009), but others predict no significant impact of metallicity for values of $[\mathrm{Fe} / \mathrm{H}]$ between -1.0 and 0.5 (Bate 2014). Observationally, Gao et al. (2014, 2017) found an inverse correlation between $[\mathrm{Fe} / \mathrm{H}]$ and multiplicity fraction in field F-, G-, and K-type dwarfs with multiple RVs from the Large Sky Area Multi-Object Fiber Spectroscopic Telescope (LAMOST, Cui et al. 2012). Hettinger et al. (2015) found the opposite trend in a smaller sample of F-type dwarfs from SEGUE. However, these studies are based on optical spectra with low resolutions $(R \sim 1800)$, which makes it hard to confidently measure small RV shifts.

Here, we address this topic with the high-resolution IR spectra from APOGEE. In Figure 11 we break down the $\Delta \mathrm{RV}_{\text {max }}$ distribution in each of the samples from Table 1 into $[\mathrm{Fe} / \mathrm{H}]$ terciles. The boundaries and median values of these terciles are shown in Figure 12. There is some variation of the tercile boundaries with $\log g$, but for the purpose of this discussion we can consider $[\mathrm{Fe} / \mathrm{H}] \lesssim-0.5$ as the low tercile, and $[\mathrm{Fe} / \mathrm{H}] \gtrsim 0.0$ as the high tercile.

In all non-RC samples from the MS/subgiants to the tip of the RGB, the $\Delta \mathrm{RV}_{\max }$ distribution in the lowest $[\mathrm{Fe} / \mathrm{H}]$ tercile is clearly above that in the highest $[\mathrm{Fe} / \mathrm{H}]$ tercile. This is true at all values of $\Delta \mathrm{RV}_{\max }$ above our $10 \mathrm{~km} \mathrm{~s}^{-1}$ threshold, provided there are enough objects to have small Poisson noise (i.e., $N_{\Delta \mathrm{RV}} V_{\max } \geqslant 10 \mathrm{~km} \mathrm{~s}^{-1} / N$ above a few times $10^{-3}$ for most of our samples). The ratio between the fraction of systems with $\Delta \mathrm{RV}_{\text {max }} \geqslant 10 \mathrm{~km} \mathrm{~s}^{-1}$ in the high and low $[\mathrm{Fe} / \mathrm{H}]$ terciles is shown in Figure 13, together with the $1 \sigma$ probability interval obtained from comparing random terciles in our Monte Carlo simulations. The ratio is roughly between 2 and 3 for all non$\mathrm{RC}$ samples except the sample at $\log g \sim 2.1$, where it reaches 4.5. The only sample that shows no significant metallicity effect is the RC. This could be related to the fact that the spread of metallicities in the RC catalog is smaller than that in the other APOGEE samples (see Figure 12). Stellar structure in the $\mathrm{RC}$ is itself sensitive to metallicity (Girardi 2016), so very-lowmetallicity He-burning stars $([\mathrm{Fe} / \mathrm{H}] \sim-1)$ will actually be on the horizontal branch instead of the RC. This introduces a selection effect that leads to a narrower $[\mathrm{Fe} / \mathrm{H}]$ distribution, where a multiplicity trend with metallicity can be easily washed out by errors in the APSCAP stellar parameters.

The most salient features of Figures 11 and 13 can be interpreted in simple terms. It is clear that low-metallicity and high-metallicity stars in APOGEE must have different multiplicity characteristics. Either the MS multiplicity fraction or the MS period distribution (or both) must be metallicity-dependent, though the clear segregation at all values of $\Delta R V_{\text {max }}$ seen in Figure 11 favors a metallicity-dependent multiplicity fraction (see discussion in Section 3 of Maoz et al. 2012). Provided that the MS period distribution remains roughly lognormal, with a sharp truncation at $P_{\text {crit }}$ for RGB stars of all metallicities, the multiplicity fraction of APOGEE targets in the lowermetallicity tercile should be a factor 2-3 higher than that in the higher -metallicity tercile, to reproduce the ratios shown in Figure 13. If $f_{m} \sim 0.35$ for the mean $([\mathrm{Fe} / \mathrm{H}] \sim-0.3)$, the multiplicity fraction for metal-poor $([\mathrm{Fe} / \mathrm{H}] \lesssim-0.5)$ stars 

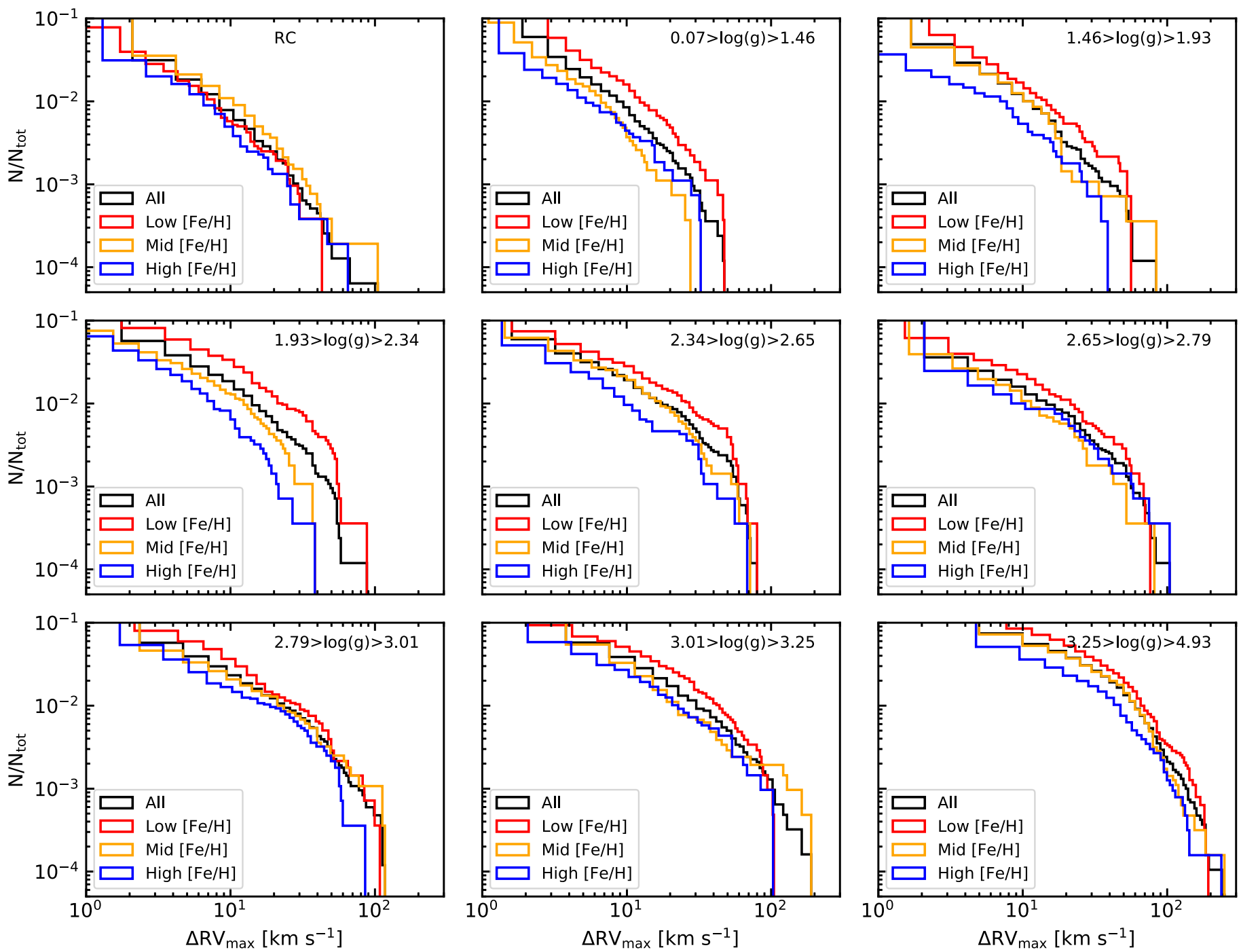

Figure 11. Cumulative histograms of $\Delta \mathrm{RV}_{\max }$ for the nine $\log g$ subsamples shown in Figure 6, broken down into [Fe/H] terciles.

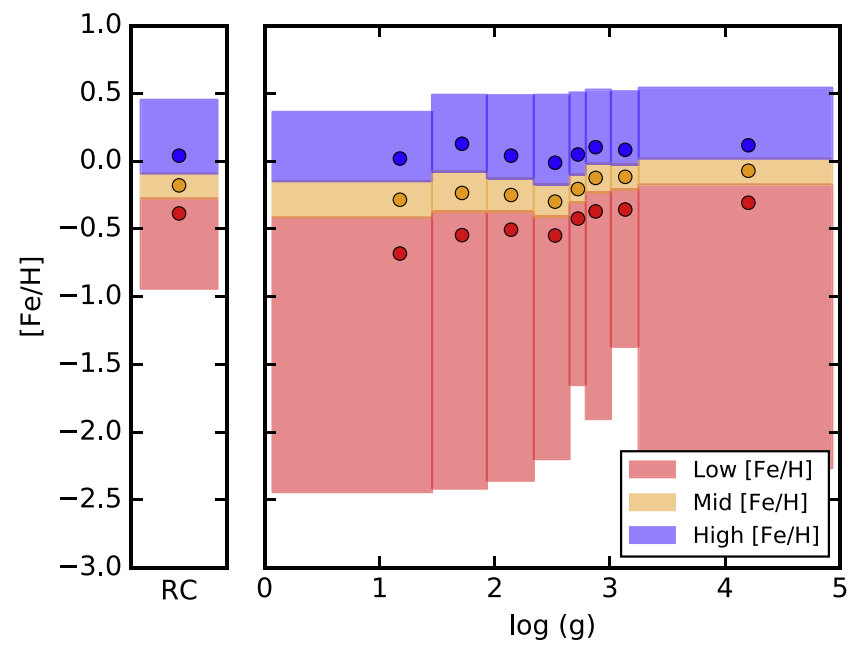

Figure 12. Location of the $[\mathrm{Fe} / \mathrm{H}]$ terciles in each $\log g$ subsample, plus the $\mathrm{RC}$. The symbols indicate the median $\log g$ of each subsample and the median $[\mathrm{Fe} / \mathrm{H}]$ of each tercile.

should be close to 0.46 , and that for metal-rich $([\mathrm{Fe} / \mathrm{H}] \gtrsim 0.0)$ stars should be close to 0.23 . The rising trend in the ratio of high $\Delta \mathrm{RV}_{\max }$ systems for $\log g \lesssim 3$ might be related to the

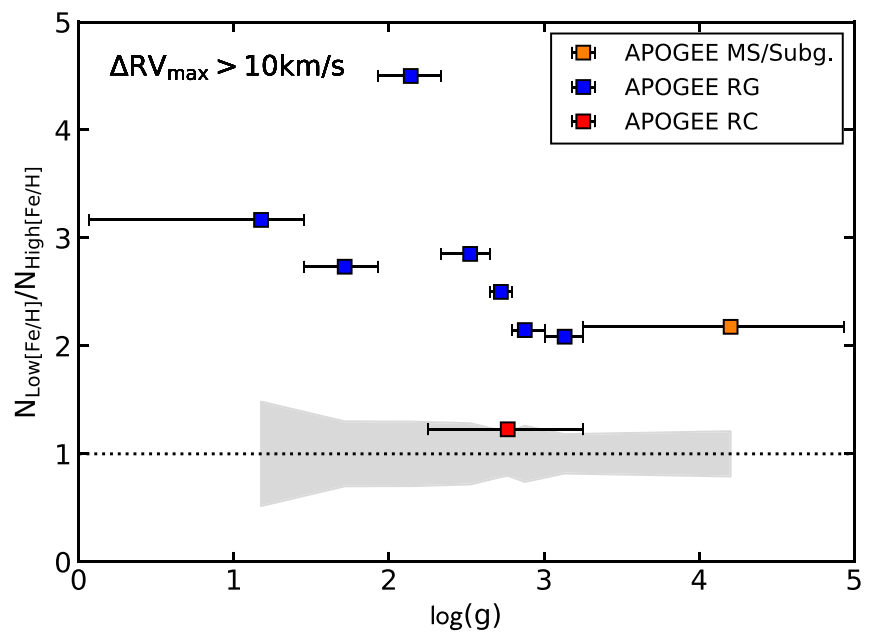

Figure 13. Ratio between the fraction of systems with $\Delta \mathrm{RV}_{\max }>10 \mathrm{~km} \mathrm{~s}^{-1}$ in the high and low $[\mathrm{Fe} / \mathrm{H}]$ terciles of each $\log g$ sample, as well as the $\mathrm{RC}$ catalog.The symbols are placed at the median $\log g$ of each sample, with the horizontal error bars giving the range of $\log g$ values. The shaded gray area around 1 is the $1 \sigma$ probability interval obtained by comparing random terciles in our Monte Carlo runs with $N=8300$. 
shift toward lower metallicity in the $[\mathrm{Fe} / \mathrm{H}]$ distributions in that $\log g$ range seen in Figure 12.

Although a metallicity-dependent multiplicity fraction does provide the simplest explanation for the APOGEE observations, we emphasize that our simple analysis can only offer a partial glimpse into what is likely a complex situation. For example, it is well known that $T_{\text {eff }}$, which we have ignored, is strongly correlated with $[\mathrm{Fe} / \mathrm{H}]$ in the RGB (see Figure 12 in Holtzman et al. 2015), with lower $[\mathrm{Fe} / \mathrm{H}]$ stars having higher $T_{\text {eff }}$ at constant $\log g$. Since $[\mathrm{Fe} / \mathrm{H}]$ is an intrinsic property of the star that is set at birth, we have chosen to present our results as a function of $[\mathrm{Fe} / \mathrm{H}]$, rather than $T_{\text {eff }}$; but see Gao et al. (2017) for a discussion of the interplay between the two parameters. Another factor that could in principle influence the $\Delta R V_{\text {max }}$ distributions is the mass of the primary, but we have found no large systematic differences in the masses determined by Ness et al. (2016) for the high- and low-metallicity terciles in our samples, so this effect, if present, must be small.

\section{Discussion}

\subsection{Multiplicity Fraction}

The best-fit value of $f_{m} \sim 0.35$ obtained by comparing our Monte Carlo simulations to the APOGEE observations of RGB stars is $\sim 60 \%$ lower than the canonical multiplicity fraction for Sun-like MS stars $(0.50 \pm 0.04$ for $\log P<8$, which is equivalent to 0.56 over the entire period range; Moe \& Di Stefano 2017). A direct comparison between these two numbers, however, is not trivial. Our code assumes the Raghavan et al. (2010) period distribution (lognormal, with a peak at $\log P \sim 5.0$, Figure 1), but the APOGEE baselines are only sensitive to systems in the tail of this distribution, below $\log P \sim 3$.3. Stellar multiplicity surveys usually have a small number of systems with such short periods, so the value of $f_{m}$ is more uncertain in this period range than the integral over all periods. After correcting the Raghavan et al. (2010) sample for completeness, Moe \& Di Stefano (2017) listed 32 systems with $\log P<3$, which implies a $\sqrt{N} / N=18 \%$ uncertainty in the value of $f_{m}$ from Poisson statistics alone, much larger than the $8 \%$ given for the entire sample. The tidal circularization model in our Monte Carlo code, which is derived from theory alone, might be inaccurate, and this could impact the number of high $\triangle \mathrm{RV}_{\text {max }}$ systems produced by our simulations. In Monte Carlo runs where the value of $P_{\text {circ }}$ shown in Figure 8 is divided by 2 and 5, we find that the relative increases in $N_{\Delta \mathrm{RV}}$ max $\geqslant 10 \mathrm{~km} \mathrm{~s}^{-1} / N$ are $\sim 3 \%$ and $\sim 5 \%$, respectively. Finally, Moe \& Di Stefano (2017) discussed the probability that the photometric primary in a SB1 binary might in fact be the mass secondary in a postcommon envelope system with a white dwarf companion (see their Section 8.3 and Figure 29). The fraction of short-period $(\log P<3.3)$ SB1 systems with white dwarf companions depends on the age of the stellar population, and is $\sim 15 \%$ at $1 \mathrm{Gyr}$ and $\sim 30 \%$ at $10 \mathrm{Gyr}$. Because the RGB stars in APOGEE have a median age of $4.6 \mathrm{Gyr}$ (Ness et al. 2016), this fraction should be around $\sim 20 \%$ for the bulk of our sample, but the impact on high-metallicity and low-metallicity stars will be different (see below). The combination of the effects we have discussed here $(\sim 18 \%$ from small sample sizes at short periods, $\sim 5 \%$ from uncertainties in the tidal circularization model, and $\sim 20 \%$ from white dwarf companions) is comparable to the $\sim 60 \%$ difference between our measurement and that of Moe \& Di Stefano (2017). Therefore, we cannot claim that our measurements of the multiplicity fraction are mutually inconsistent.

\subsection{Metallicity Effect}

The metallicity trend shown in Figures 11 and 13 will also be affected by the presence of white dwarf companions to shortperiod SB1 binaries. However, the increase of the fraction of white dwarf companions from $15 \%$ to $30 \%$ between highmetallicity and low-metallicity populations estimated by Moe \& Di Stefano (2017) cannot explain the factor 2-3 increase in the fraction of high $\Delta \mathrm{RV}_{\max }$ systems seen in APOGEE. In principle, a systematic difference between the masses of stars in the high- and low-metallicity terciles of each $\log g$ sample could explain our results, but this difference would have to be very large. If the close $(\log P \lesssim 4)$ binary fraction scales as $M^{0.7}$ (Moe \& Di Stefano 2017), the median mass of the stars in the low-metallicity terciles would have to be a factor $\sim 2.6$ higher than the median mass of the stars in the high-metallicity terciles to increase the fraction of systems with $\Delta R V_{\max }>$ $10 \mathrm{~km} \mathrm{~s}^{-1}$ by a factor of 2 . In our RGB samples, the median masses of stars in the high- and low-metallicity terciles measured by Ness et al. (2016) vary by a few tenths of a $M_{\odot}$ at most.

We conclude that the inverse correlation between metallicity and multiplicity fraction seen in APOGEE must be real. Our result is in qualitative agreement with the works of Gao et al. $(2014,2017)$ that used low-resolution spectra of MS stars, and the findings of Yuan et al. (2015) from photometric studies, but it is in apparent contradiction with the results of Hettinger et al. (2015) from nearby F-type dwarfs. Moe \& Di Stefano (2013) determined that the binary fraction at very short periods $(\log P<1.3)$ for $10 M_{\odot}$ primaries does not vary by more than $\sim 30 \%$ across the $[\mathrm{Fe} / \mathrm{H}]$ range between -0.7 and 0.1 by studying early-type eclipsing binaries in the Milky Way and the Magellanic Clouds. Older studies based on small samples of spectroscopic orbits by Latham et al. (2002) and Carney et al. (2005) also reported no significant correlation between metallicity and multiplicity. However, caution must be used again when comparing such different surveys. Because they are not looking at the same stars, and they are not sensitive to the same periods, it is hard to establish to what degree these observational studies might be mutually inconsistent. To date, ours is the only study based on high-resolution spectra that span all evolutionary phases between the MS and core Heburning for Sun-like stars in a large fraction of the Milky Way disk, with a sample size orders of magnitude larger than any previous attempt. The recent re-analysis of the MS spectra in APOGEE by El-Badry et al. (2018b) has provided an independent confirmation of the inverse correlation between metallicity and multiplicity that we report here.

A detailed interpretation of the metallicity effect that we have discovered is outside the scope of the present work. Naively, one might say that our results support the conclusions of Machida (2008) and Machida et al. (2009) regarding the impact of metallicity on star formation, and that studies where metallicity has little or no effect on stellar multiplicity at birth can be ruled out. (e.g., Bate 2014). These multiplicity statistics set "at birth" will be subject to dynamical evolution through orbital capture and disruption, which can be quite different in different environments such as the thin disk, the thick disk, and the halo. However, numerical simulations imply that the hard binaries we study here $(\log P \leqslant 3.3)$ should be relatively 
unaffected by this (Kroupa \& Burkert 2001; Goodwin \& Kroupa 2005; Kroupa \& Petr-Gotzens 2011). More complex processes involving stellar mergers can also affect the relationship between multiplicity, metallicity, and stellar age (e.g., Jofré et al. 2016; Izzard et al. 2018).

Regardless of its origin, a metallicity-dependent multiplicity fraction has profound implications for the rates of interacting binaries like SNe Ia and neutron star mergers, which are observed in host galaxies with a wide range of redshifts and therefore metallicities (Zahid et al. 2013). Binary population synthesis calculations for these objects often assume that the multiplicity statistics in the MS (i.e., the initial conditions) are not metallicity-dependent (e.g., Claeys et al. 2014; de Mink \& Belczynski 2015), an assumption that should be revised in light of our results. The dynamical stability of circumbinary planets will also be affected by a higher fraction of short-period binaries at low metallicities (Jaime et al. 2014), which could be related to the well-established paucity of planets around lowmetallicity stars (Johnson et al. 2010).

\subsubsection{Future Prospects}

For the first time, APOGEE has offered us a panoramic view of the interplay between stellar multiplicity and stellar evolution across the Milky Way as stars leave the MS, climb the RGB, and settle into the RC after He ignition. This interplay had been previously constrained by indirect means (e.g., stellar rotation and asteroseismology; Tayar et al. 2015), but our analysis of the sparsely sampled RV curves from APOGEE allows us to examine it directly and with a large statistical sample. The attrition of short-period companions as stars undergo RLOF during this process must be rapid and efficient, because the APOGEE $\triangle \mathrm{RV}_{\text {max }}$ measurements are consistent with no systems with $P<P_{\text {crit. }}$. Future work on this data set will allow us to quantify this process and estimate a rate of RLOF events due to unstable mass transfer during $\mathrm{H}$-shell burning in the disk of the Milky Way. The fate of the systems that are removed from the APOGEE sample is a matter of considerable interest. For mass donors with convective envelopes, like the APOGEE RGB stars, mass transfer is unstable over a large range in mass ratios (Pavlovskii \& Ivanova 2015), and the outcome should be a common envelope episode in the vast majority of cases. A few of these episodes have now been observed as optical transients like V838 Mon and V1309 Sco (Ivanova et al. 2013), allowing us to constrain the Galactic rate of stellar mergers to $\sim 0.5 \mathrm{yr}^{-1}$, albeit with large error bars (Kochanek et al. 2014). The few epoch APOGEE spectra can shed light on the fraction of common envelope episodes that are associated with such transients.

\section{Conclusions}

We have presented a statistical study of stellar multiplicity that takes advantage of the high resolution and exceptional depth achieved by the APOGEE IR spectrographs. We defined a sample of $\sim 90,000$ stars in the field of the Milky Way with two or more high-quality RV measurements from APOGEE, spanning the evolutionary stages between the MS and core $\mathrm{He}$ burning. We used the maximum RV shift in these stars, $\Delta \mathrm{RV}_{\max }$, as a figure of merit to study stellar multiplicity, and we established that stars with $\Delta \mathrm{RV}_{\max } \geqslant 10 \mathrm{~km} \mathrm{~s}^{-1}$ are bona fide binaries, unaffected by measurement errors. Given the distribution of APOGEE temporal baselines, we are sensitive to systems with $\log P \leqslant 3.3$ (5.5 years). We found a strong correlation between the maximum value of $\Delta \mathrm{RV}_{\max }$ and $\log g$, with MS stars having $\Delta \mathrm{RV}_{\max }$ as high as $\sim 300 \mathrm{~km} \mathrm{~s}^{-1}$ and stars close to the tip of the RGB only going as high as $\sim 30 \mathrm{~km} \mathrm{~s}^{-1}$. This effect is accompanied by an attrition of bona fide binaries at all values of $\Delta \mathrm{RV}_{\max }$ as stars climb up the $\mathrm{RGB}$. The $\Delta \mathrm{RV}_{\text {max }}$ distribution of core He-burning RC stars is similar to that of stars close to the tip of the RGB. Finally, we found a strong correlation between metallicity and $\Delta \mathrm{RV}_{\max }$, with metal-poor stars in APOGEE $([\mathrm{Fe} / \mathrm{H}] \lesssim-0.5)$ being more likely to have any $\Delta \mathrm{RV}_{\max }$ values above our $10 \mathrm{~km} \mathrm{~s}^{-1}$ threshold than metal-rich stars $([\mathrm{Fe} / \mathrm{H}] \gtrsim 0.5)$.

We used simple Monte Carlo simulations to interpret these observations, and found that the attrition of high $\Delta R V_{\text {max }}$ systems with decreasing $\log g$ is consistent with the sharp truncation of a lognormal MS period distribution like that measured by Raghavan et al. (2010) for Sun-like dwarfs in the solar neighborhood at the critical period for RLOF for each $\log g$. For the RGB stars with $M \sim 1 M_{\odot}$ that form the bulk of the APOGEE sample and are best characterized by the APSCAP pipeline, we find that a multiplicity fraction of 0.35 matches the observations quite well. The correlation between $\Delta R V_{\text {max }}$ and metallicity can be explained with a metallicitydependent multiplicity fraction, with metal-poor stars having a factor 2-3 more close companions than metal-rich stars. This has profound implications for the formation rates of interacting binaries observed by astronomical transient surveys and gravitational wave detectors, as well as the habitability of circumbinary planets.

Jieun Choi kindly made details of her MIST models available to us upon request. Robert Mathieu made useful suggestions that helped improve this manuscript. This research was supported in part by Scialog Scholar grant 24215 from the Research Corporation. C.A.P. is thankful to the Spanish MINECO for funding under grant AYA2014-56359-P. T.C. B. acknowledges partial support from grant PHY 14-30152: Physics Frontier Center/JINA Center for the Evolution of the Elements (JINA-CEE), awarded by the US National Science Foundation. This research has made use of NASA's Astrophysics Data System.

Funding for the Sloan Digital Sky Survey IV has been provided by the Alfred P. Sloan Foundation, the U.S. Department of Energy Office of Science, and the Participating Institutions. SDSS-IV acknowledges support and resources from the Center for High-Performance Computing at the University of Utah. The SDSS web site is www.sdss.org.

SDSS-IV is managed by the Astrophysical Research Consortium for the Participating Institutions of the SDSS Collaboration including the Brazilian Participation Group, the Carnegie Institution for Science, Carnegie Mellon University, the Chilean Participation Group, the French Participation Group, Harvard-Smithsonian Center for Astrophysics, Instituto de Astrofísica de Canarias, The Johns Hopkins University, Kavli Institute for the Physics and Mathematics of the Universe (IPMU)/University of Tokyo, Lawrence Berkeley National Laboratory, Leibniz Institut für Astrophysik Potsdam (AIP), Max-Planck-Institut für Astronomie (MPIA Heidelberg), Max-Planck-Institut für Astrophysik (MPA Garching), MaxPlanck-Institut für Extraterrestrische Physik (MPE), National Astronomical Observatories of China, New Mexico State University, New York University, University of Notre Dame, 
Observatário Nacional/MCTI, The Ohio State University, Pennsylvania State University, Shanghai Astronomical Observatory, United Kingdom Participation Group, Universidad Nacional Autónoma de México, University of Arizona, University of Colorado Boulder, University of Oxford, University of Portsmouth, University of Utah, University of Virginia, University of Washington, University of Wisconsin, Vanderbilt University, and Yale University.

Software: Ipython (Perez \& Granger 2007), Astropy (Robitaille et al. 2013), Matplotlib (Hunter 2007).

\section{ORCID iDs}

Carles Badenes (16) https://orcid.org/0000-0003-3494-343X

Kevin Covey (ib https://orcid.org/0000-0001-6914-7797

Matthew G. Walker (1) https://orcid.org/0000-0003-2496-1925

Nicholas Troup (1) https://orcid.org/0000-0003-3248-3097

David Nidever (1) https://orcid.org/0000-0002-1793-3689

Brett Andrews (1) https://orcid.org/0000-0001-8085-5890

Timothy C. Beers (1) https://orcid.org/0000-0003-4573-6233

Jo Bovy (ib https://orcid.org/0000-0001-6855-442X

Steven R. Majewski i https://orcid.org/0000-0003-

2025-3147

Marc Pinsonneault (i) https://orcid.org/0000-0002-7549-7766

Keivan G. Stassun (1) https://orcid.org/0000-0002-3481-9052

Guy S. Stringfellow (1) https://orcid.org/0000-0003-1479-3059

\section{References}

Albareti, S., Allende Prieto, C., Almeida, A., et al. 2017, ApJS, 233, 25 Andrews, J. J., Agüeros, M., Brown, W. R., et al. 2016, ApJ, 828, 38 Badenes, C., \& Maoz, D. 2012, ApJL, 749, L11

Bate, M. R. 2014, MNRAS, 442, 285

Blanton, M. R., Bershady, M. A., Abolfathi, B., et al. 2017, AJ, 154, 28

Bovy, J., Nidever, D., Hix, H.-W., et al. 2014, ApJ, 790, 127

Carney, B. W., Aguilar, L. A., Latham, D. W., \& Laird, J. B. 2005, AJ, 129, 1886

Carney, B. W., Latham, D. W., Stefanik, R. P., Laird, J. B., \& Morse, J. A. 2003, AJ, 125, 293

Chini, R., Fuhrmann, K., Barr, A., et al. 2014, MNRAS, 437, 879

Choi, J., Dotter, A., Conroy, C., et al. 2016, ApJ, 823, 102

Claeys, J. S. W., Pols, O. R., Izzard, R. G., Vink, J., \& Verbunt, F. W. M. 2014, A\&A, 563, A83

Cottaar, M., Covey, K. R., Meyer, M. R., et al. 2014, ApJ, 794, 125

Cui, X.-Q., Zhao, Y.-H., Chu, Y.-Q., et al. 2012, RAA, 12, 1197

de Mink, S. E., \& Belczynski, K. 2015, ApJ, 814, 58

Duchêne, G., \& Kraus, A. 2013, ARA\&A, 51, 269

Duquennoy, A., \& Mayor, M. 1991, A\&A, 248, 485

Eggleton, P. P. 1983, ApJ, 268, 368

El-Badry, K., Rix, H.-W., Ting, Y.-S., et al. 2018a, MNRAS, 473, 5043

El-Badry, K., Ting, Y.-S., Rix, H.-W., et al. 2018b, MNRAS, in press

Fernandez, M. A., Covey, K. R., De Lee, N., et al. 2017, PASP, 978, 084201

Gao, S., Liu, C., Zhang, X., et al. 2014, ApJL, 788, L37

Gao, S., Zhao, H., Yang, H., \& Gao, R. 2017, MNRAS, 469, L68

García Pérez, A. E., Allende Prieto, C., Holtzman, J. A., et al. 2016, AJ, 151,144

Geller, A. M., \& Mathieu, R. D. 2012, AJ, 144, 54

Geller, A. M., Mathieu, R. D., Harris, H. C., \& McClure, R. D. 2008, AJ, 135,2264

Girardi, L. 2016, ARA\&A, 54, 95
Goodwin, S. P., \& Kroupa, P. 2005, A\&A, 439, 565

Gosnell, N. M., Mathieu, R. D., Geller, A. M., et al. 2015, ApJ, 814, 163

Gunn, J. E., Siegmund, W. A., Mannery, E. J., et al. 2006, AJ, 131, 2332

Hekker, S., Snellen, I. A. G., Aerts, C., et al. 2008, A\&A, 480, 215

Hettinger, T., Badenes, C., Strader, J., Bickerton, S. J., \& Beers, T. C. 2015, ApJL, 806, L2

Holtzman, J. A., Shetrone, M., Johnson, J. A., et al. 2015, AJ, 150, 148

Hunter, J. D. 2007, CSE, 9, 90

Ivanova, N., Justham, S., Avendano Nandez, J. L., \& Lombardi, J. C. 2013, Sci, 339, 433

Izzard, R. G., Preece, H., Jofre, P., et al. 2018, MNRAS, 473, 2984

Jaime, L. G., Aguilar, L., \& Pichardo, B. 2014, MNRAS, 443, 260

Jofré, P., Jorissen, A., Van Eck, S., et al. 2016, A\&A, 595, A60

Johnson, J. A., Aller, K. M., Howard, A. W., \& Crepp, J. R. 2010, PASP, 122,905

Kochanek, C. S., Adams, S. M., \& Belczynski, K. 2014, MNRAS, 443, 1319

Kroupa, P., \& Burkert, A. 2001, ApJ, 555, 945

Kroupa, P., \& Petr-Gotzens, M. G. 2011, A\&A, 529, A92

Lada, C. J. 2006, ApJL, 640, L63

Latham, D. W., Stefanik, R. P., Torres, G., et al. 2002, AJ, 124, 1144

Leiner, E. M., Mathieu, R. D., Gosnell, N. M., \& Geller, A. M. 2015, AJ, 150,10

Machida, M. N. 2008, ApJL, 682, L1

Machida, M. N., Omukai, K., Matsumoto, T., \& Inutsuka, S.-i. 2009, MNRAS, 399, 1255

Majewski, S. R., Schiavon, R. P., Frinchaboy, P. M., et al. 2017, AJ, 154, 94

Maoz, D., Badenes, C., \& Bickerton, S. J. 2012, ApJ, 751, 143

Maoz, D., \& Hallakoun, N. 2016, MNRAS, 467, 1414

Maoz, D., Mannucci, F., \& Nelemans, G. 2014, ARA\&A, 52, 107

Matijevic, G., et al. 2011, AJ, 141, 200

Mazeh, T. 2008, in EAS Publ Ser. 29, Tidal Effects in Stars, Planets and Disks', ed. M.-J. Goupil \& J.-P. Zahn (les Ulis: EDP Sciences), 1

Meibom, S., \& Mathieu, R. D. 2005, ApJ, 620, 970

Melo, C. H. F. 2003, A\&A, 410, 269

Merle, T., van Eck, S., Jorissen, A., et al. 2017, A\&A, 608, A95

Moe, M., \& Di Stefano, R. 2013, ApJ, 778, 95

Moe, M., \& Di Stefano, R. 2017, ApJS, 230, 15

Ness, M., Hogg, D. W., Rix, H.-W., et al. 2016, ApJ, 823, 114

Ness, M., Hogg, D. W., Rix, H.-W., Ho, A. Y. Q., \& Zasowski, G. 2015, ApJ, 808,16

Nidever, D., Holtzman, J. A., Allende Prieto, C., et al. 2015, AJ, 150, 173

Pavlovskii, K., \& Ivanova, N. 2015, MNRAS, 449, 4415

Paxton, B., Bildsten, L., Dotter, A., et al. 2011, ApJS, 192, 3

Paxton, B., Cantiello, M., Arras, P., et al. 2013, ApJS, 208, 4

Paxton, B., Marchant, P., Schwab, J., et al. 2015, ApJS, 220, 15

Perez, F., \& Granger, B. E. 2007, CSE, 9, 21

Pinsonneault, M. H., Elsworth, Y., Epstein, C., et al. 2014, ApJS, 215, 19

Price-Jones, N., \& Bovy, J. 2018, MNRAS, 475, 1410

Price-Whelan, A. M., Hogg, D. W., Foreman-Mackey, D., \& Rix, H.-W. 2017, ApJ, 837, 20

Raghavan, D., McAlister, H. A., Henry, T. J., et al. 2010, ApJS, 190,

Robitaille, T. P., Tollerund, E. J., Greenfield, P., et al. 2013, A\&A, 558, A33

Sana, H., de Mink, S. E., de Koter, A., et al. 2012, Sci, 337, 444

Shporer, A., Fuller, J., Isaacson, H., et al. 2016, ApJ, 829, 34

Tayar, J., Ceillier, T., García-Hernández, D. A., et al. 2015, ApJ, 807, 82

Tokovinin, A., Thomas, S., Sterzik, M., \& Udry, S. 2006, A\&A, 450, 681

Troup, N. W., Nidever, D. L., De Lee, N., et al. 2016, AJ, 151, 85

Verbunt, F., \& Phinney, E. 1995, A\&A, 296, 709

Walker, M. G., Olszewski, E. W., \& Mateo, M. 2015, MNRAS, 448, 2717

Yanny, B., Rockosi, C., Newberg, H. J., et al. 2009, AJ, 137, 4377

Yuan, H., Liu, X., Xiang, M., et al. 2015, ApJ, 799, 135

Zahid, H. J., Geller, M. J., Kewley, L. J., et al. 2013, ApJL, 771, L19

Zahn, J.-P. 1989, A\&A, 220, 112

Zasowski, G., Johnson, J. A., Frinchaboy, P. M., et al. 2013, AJ, 146, 81 